\title{
99m-Tc-Labeled Superparamagnetic Iron Oxide Nanoparticles for Multimodality SPECT/MRI of Sentinel Lymph Nodes
}

\author{
Renata Madru ${ }^{1}$, Pontus Kjellman ${ }^{1,2}$, Fredrik Olsson ${ }^{2}$, Karin Wingårdh ${ }^{1}$, Christian Ingvar ${ }^{3}$, Freddy Ståhlberg ${ }^{1,4}$, \\ Johan Olsrud $^{5}$, Jimmy Lätt ${ }^{5}$, Sarah Fredriksson ${ }^{2}$, Linda Knutsson ${ }^{1}$, and Sven-Erik Strand ${ }^{1,4}$ \\ ${ }^{1}$ Department of Medical Radiation Physics, Lund University, Lund, Sweden; ${ }^{2}$ Genovis AB, Lund, Sweden; ${ }^{3}$ Department of Surgery, \\ Lund University, Lund, Sweden; ${ }^{4}$ Lund University Bioimaging Center (LBIC), Lund, Sweden; and ${ }^{5}$ Center for Medical Imaging and \\ Physiology, Skane University Hospital, Lund, Sweden
}

The purpose of this study was to develop multimodality SPECT/ MRI contrast agents for sentinel lymph node (SLN) mapping in vivo. Methods: Nanoparticles with a solid iron oxide core and a polyethylene glycol coating were labeled with ${ }^{99 m T c}$. The labeling efficiency was determined with instant thin-layer chromatography and magnetic separation. The stability of the radiolabeled superparamagnetic iron oxide nanoparticles (SPIONs) was verified in both sterile water and human serum at room temperature 6 and $24 \mathrm{~h}$ after labeling. Five Wistar rats were injected subcutaneously in the right hind paw with ${ }^{99 m T c-S P I O N s ~(25-50 ~ M B q, ~}$ $\sim 0.2 \mathrm{mg}$ of $\mathrm{Fe}$ ) and sacrificed $4 \mathrm{~h}$ after injection. Two animals were imaged with SPECT/MRI. All 5 rats were dissected; the lymph nodes, liver, kidneys, spleen, and hind paw containing the injection site were removed and weighed; and activity in the samples was measured. The microdistribution within the lymph nodes was studied with digital autoradiography. Results: The efficiency of labeling of the SPIONs was $99 \% 6 \mathrm{~h}$ after labeling in both water and human serum. The labeling yield was $98 \%$ in water and $97 \%$ in human serum $24 \mathrm{~h}$ after labeling. The SLN could be identified in vivo with SPECT/MRI. The accumulation of 99mTc-SPIONs (as the percentage injected dose/g [\%ID/g]) in the SLN was $100 \%$ $\mathrm{ID} / \mathrm{g}$, whereas in the liver and spleen it was less than $2 \% \mathrm{ID} / \mathrm{g}$. Digital autoradiography images revealed a nonhomogeneous distribution of $99 \mathrm{mTC}$-SPIONs within the lymph nodes; nanoparticles were found in the cortical, subcapsular, and medullary sinuses. Conclusion: This study revealed the feasibility of labeling SPIONs with $99 \mathrm{mT}$. The accumulation of $99 \mathrm{mTc}$-SPIONs in lymph nodes after subcutaneous injection in animals, verified by SPECT/MRI, is encouraging for applications in breast cancer and malignant melanoma.

Key Words: SPIONs; iron oxide; nanoparticles; 99mTc; SPECT/ MRI; lymph nodes

J Nucl Med 2012; 53:459-463

DOI: 10.2967/jnumed.111.092437

\footnotetext{
Received Jun. 17, 2011; revision accepted Oct. 17, 2011.

For correspondence or reprints contact: Renata Madru, Department of Medical Radiation Physics, Lund University, Skane University Hospital, SE22185 Lund, Sweden.

E-mail: Renata.Madru@med.lu.se

Published online Feb. 9, 2012.

COPYRIGHT @ 2012 by the Society of Nuclear Medicine, Inc.
}

$\mathbf{T}$ he sentinel lymph node (SLN) is defined as the first regional lymph node receiving lymphatic drainage from a malignant tumor (1) and the first node to which metastatic cells are likely to anchor. Therefore, accurate detection and characterization of the SLN is of major importance for cancer staging and for the choice of therapy in patients with breast cancer and malignant melanoma. The current gold standard relies on lymphoscintigraphy after intradermal injection of radiolabeled colloids and blue dye to intraoperatively identify the SLN by dissection and histopathologic examination (2). The radiopharmaceuticals most frequently used for SLN imaging are ${ }^{99 \mathrm{~m} T c-l a b e l e d ~ c o l l o i d s ~ a n d ~ m a c-~}$ romolecules such as trisulfide, dextran, and human serum albumin (3-5). The current technique, however, is limited because of the nonspecificity of the tracer and the lack of anatomic information in scintigraphic images. Preoperative planning and identification of the SLN often rely on the experience of the surgeon.

We propose combining information from high-resolution MRI and high-sensitivity SPECT images to provide more accurate and less invasive identification of the SLN before surgery. The use of radioactivity would help to identify the SLN, and MRI in multiple planes with submillimeter resolution would provide better presurgical information about the location and characterization of the node. During the past decade, much research has been focused on finding alternative carriers (such as nanocarriers based on quantum dots, liposomes, ultrasound nanobubbles, or magnetic nanoparticles) to provide less invasive but more accurate localization of the SLN (6). Although the toxicity and biocompatibility of quantum dots have not yet been fully evaluated, magnetic nanoparticles have been in clinical use since the 1960s-for example, for the treatment of iron deficiency anemia (7). Superparamagnetic iron oxide nanoparticles (SPIONs) consist of a magnetic core surrounded by a biocompatible coating material, often dextran or polyethylene glycol. SPION-based contrast agents exhibit high relaxivity in MRI. SPIONs cause shortening of the transverse relaxation of the spins and local inhomogeneity in the magnetic field; consequently, the signal intensity in tissues containing contrast agents is significantly 
decreased. The signal intensity loss (or negative contrast enhancement) is dose dependent, and an appropriate pulse sequence is required.

Contrast agents containing SPIONs have shown great potential for identifying metastases in the reticuloendothelial system, including the liver, spleen, bone marrow, and lymph nodes (8-11). Additionally, SPIONs can serve as a platform for other functional moieties, including radionuclides.

The aims of this study were to develop and evaluate new contrast agents based on biocompatible SPIONs and to label them with ${ }^{99 \mathrm{~m}} \mathrm{Tc}$ for multimodality imaging with SPECT/ MRI of the SLN, the goal being to provide high-sensitivity, morphologic images with good soft-tissue contrast.

\section{MATERIALS AND METHODS}

\section{Nanoparticles}

Characteristics. The SPIONs (Genovis AB) used in this study were designed for application in the fields of biomedicine and biotechnology. The monodisperse core of the nanoparticle measures $11 \mathrm{~nm}(\mathrm{SD}, 2 \mathrm{~nm})$, as determined by transmission electron microscopy (TEM), is composed of $\mathrm{Fe}_{3} \mathrm{O}_{4}$, and has a high level of magnetic saturation $(\sim 80$ electromagnetic units/g). The solid iron oxide core is coated with functionalized polyethylene glycol about 3 $\mathrm{nm}$ thick. The coated particle has a hydrodynamic diameter of $18 \mathrm{~nm}$ $(\mathrm{SD}, 3 \mathrm{~nm})$. The coating is biocompatible, prevents aggregation, and ensures that the particles can be easily labeled with other moieties.

Labeling. SPIONs in a sterile water suspension (a volume of $0.6 \mathrm{~mL}$ containing $\mathrm{Fe}$ at $3.4 \mathrm{mg} / \mathrm{mL}$ ) were labeled with ${ }^{99 \mathrm{~m}} \mathrm{Tc}-$

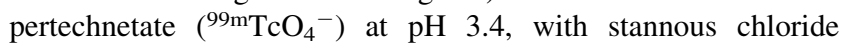
$\left(\mathrm{SnCl}_{2} \cdot 2 \mathrm{H}_{2} \mathrm{O}\right)$ as a reducing agent. ${ }^{99 \mathrm{~m}_{\mathrm{TcO}}}{ }^{-}$at $500-900 \mathrm{MBq} /$ $0.7 \mathrm{~mL}$, eluted from a ${ }^{99} \mathrm{Mo} / 99 \mathrm{~m} \mathrm{Tc}$ generator (DRN 4329; Ultratechnekow FM), was injected into a sterile vacuum collection vial. Subsequently, $0.5 \mathrm{mg}$ of $\mathrm{SnCl}_{2} \cdot 2 \mathrm{H}_{2} \mathrm{O}$ was dissolved in $0.5 \mathrm{~mL}$ of distilled water, and the mixture was stirred for a few seconds and passed through a $0.22-\mu \mathrm{m}$ Millipore filter into the vial containing the ${ }^{99} \mathrm{TcO}_{4}{ }^{-}$. SPIONs were added to the vial, and the vial was gently shaken for $30 \mathrm{~s}$. The mixture was allowed to incubate for $60 \mathrm{~min}$ at room temperature.

Quality Control. The labeling efficiency was determined with 2 methods. The first, instant thin-layer chromatography (ITLC), was performed on silica gel-impregnated glass fiber sheets $(5 \times 15 \mathrm{~cm}$; Life Science) with $85 \%$ aqueous methanol as the mobile phase (12). The sheets were dried and cut into $1-\mathrm{cm}$ segments, and radioactivity was measured in an $\mathrm{NaI}$ well-type detector (1282 CompuGamma CS; LKB Wallac). In the second method, the labeling efficiency was evaluated by separating the ${ }^{99 \mathrm{~m}} \mathrm{Tc}-\mathrm{SPIONs}$ from the buffer with MACS MS columns (Miltenyi Biotech) and a magnet (Miltenyi Biotech). The activity of the ${ }^{99 \mathrm{~m}}$ Tc-SPIONs was compared with the activity of the buffer by use of a dose calibrator (VDC 303; Veenstra Instruments). The colloidal stability of the ${ }^{99 \mathrm{~m}} \mathrm{Tc}-\mathrm{SPIONs}$ in sterile water $(\mathrm{pH} 7)$ and in human serum ( $\mathrm{pH} 7$ ) was verified with ITLC 6 and $24 \mathrm{~h}$ after labeling.

The sizes of the SPIONs and ${ }^{99 \mathrm{~m}} \mathrm{Tc}-\mathrm{SPIONs}$ were measured by TEM (Philips CM 10 system). Multiple samples of unlabeled and ${ }^{99 \mathrm{~m}}$ Tc-labeled nanoparticles were spotted onto carbon grids of 400 mesh. The particle diameter was determined as the mean of 5 cross-sectional measurements. The images were compared visually to identify discrepancies-such as the coating or shape of the SPIONs-between unlabeled and ${ }^{99 \mathrm{~m}} \mathrm{Tc}$-labeled nanoparticles.
To characterize the ${ }^{99 \mathrm{~m}} \mathrm{Tc}-\mathrm{SPIONs}$ under physiologic conditions, we measured the $\zeta$-potential (Malvern Zetasizer Nano with clear disposable cells; Malvern Instruments) in 5 solutions of ${ }^{99 \mathrm{~m}}$ Tc-SPIONs with $\mathrm{pH}$ values ranging from 4 to 9 . The $\mathrm{pH}$ was adjusted with acetate, 2-( $N$-morpholino)ethanesulfonic acid, $\mathrm{N}$-2hydroxyethylpiperazine- $N^{\prime}$-2-ethanesulfonic acid, and carbonate solutions (Sigma Aldrich).

\section{Animal Studies}

Imaging. To evaluate the biodistribution of the ${ }^{99 \mathrm{~m} T c-S P I O N s}$ in vivo, we used 5 white Wistar male rats (Charles River) weighing 230-250 g. The study was performed in compliance with national and local regulations for the conduct of animal experiments. The animals were lightly anesthetized with isoflurane, and ${ }^{99 \mathrm{~m}}$ Tc-SPIONs (25-50 MBq, equivalent to $0.05-0.07 \mathrm{~mL} ; \sim 0.2$ $\mathrm{mg}$ of Fe; $\mathrm{pH} 3.4$ ) were subcutaneously injected in the right hind paw. The animals were returned to their cages and were sacrificed by inhalation of $\mathrm{CO}_{2}$ after $4 \mathrm{~h}$. Two of the 5 rats were used for SPECT/MRI.

Static anterior and posterior images were collected for $15 \mathrm{~min}$ with a scintillation camera (GE Hawkeye; GE Healthcare) equipped with a low-energy, high-resolution parallel-hole collimator. SPECT/CT projections were collected for $1 \mathrm{~h}$, at 60 s/projection, with a $20 \%$ energy window centered at $140 \mathrm{keV}$.

The same 2 animals were then imaged with a 3-T MRI camera (Philips Achieva, equipped with a Sense-Flex-M coil). A T2weighted spin-echo (SE) pulse sequence was acquired to visualize the anatomic and morphologic details. A gradient-echo (GRE) pulse sequence was used for T2*-weighted imaging of the lymph nodes. The SE imaging parameters were as follows: TE/TR, 80/ 3,000 ; field of view, $100 \mathrm{~mm}$; voxel size, $0.5 \times 0.5 \times 1.3 \mathrm{~mm}^{3}$; flip angle, $90^{\circ}$; and number of signals averaged, 10 . The GRE imaging parameters were as follows: TE/TR, 14.6/2,000; voxel size, $0.8 \times$ $0.8 \times 1.3 \mathrm{~mm}^{3}$; flip angle, $90^{\circ}$; and number of signals averaged, 10. The signal intensity of the SLN on the injected side of the animal (containing the ${ }^{99 \mathrm{~m}} \mathrm{Tc}-\mathrm{SPIONs}$ ) was visually compared with that of the node on the other side.

Biodistribution. All 5 animals were dissected; the lymph nodes, kidneys, spleen, liver, and hind paw containing the injection site were removed and weighed; and radioactivity was measured in the NaI well-type detector. All measurements were corrected for background and radioactive decay. The accumulation of the ${ }^{99 \mathrm{~m} T c-S P I O N s}$ in the dissected samples was expressed as the percentage injected dose/g of wet tissue (\% ID/g).

To study the distribution of the ${ }^{99 \mathrm{~m}}$ Tc-SPIONs within the SLN, we froze the popliteal and lumbar lymph nodes on dry ice. Consecutive $100-\mu \mathrm{m}$ coronal sections were obtained with a cryostat (Microme, Vacutome HM $500 \mathrm{OM}$; Microme International) mounted on microscope slides, and air dried at room temperature. The sections were imaged by digital autoradiography (DARG) with a silicon strip detector (Biomolex 700 Imager; Biomolex AS).

\section{RESULTS}

\section{Radiolabeling}

The radiolabeling efficiency, as determined by ITLC, was $99 \%$. The radiolabeled SPIONs remained at the origin on the sheets, whereas free ${ }^{99} \mathrm{mcO}_{4}{ }^{-}$moved with the solvent front at an $\mathrm{R}_{\mathrm{f}}$ value of 0.7 . The amount of free ${ }^{99} \mathrm{TcO}_{4}{ }^{-}$in a sterile water suspension or in human serum $6 \mathrm{~h}$ after labeling was less than $1 \%$. Similar results were 

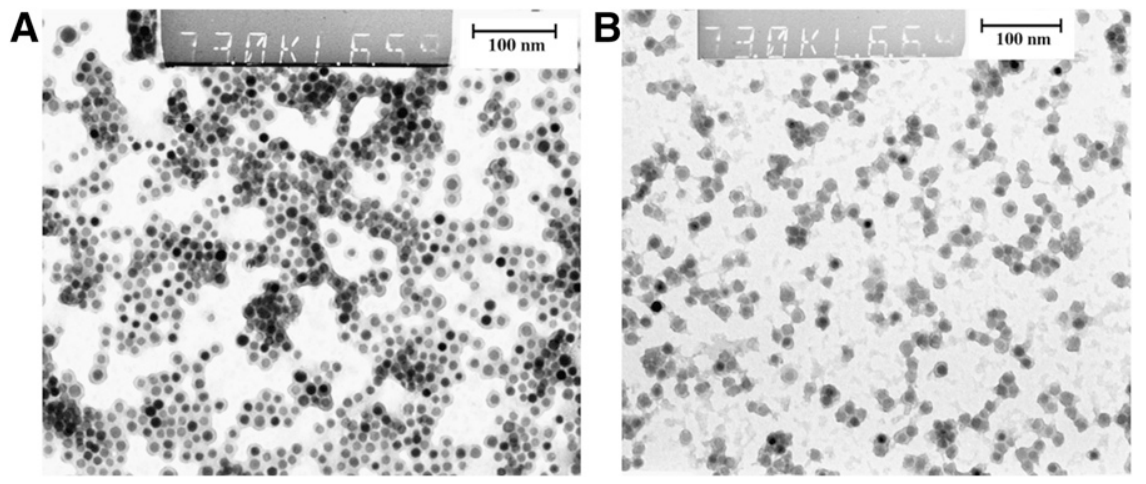

FIGURE 1. TEM images of SPIONs (A) and 99mTc-SPIONs (B) at $73 \mathrm{MV}$. Mean diameter of nanoparticles in both preparations was $13 \mathrm{~nm}(\mathrm{SD}, 2 \mathrm{~nm})$, demonstrating that labeling had no adverse effect.

obtained with the magnetic separation method. As determined by ITLC, the stability of the ${ }^{99 \mathrm{~m}} \mathrm{Tc}$-SPIONs at room temperature $24 \mathrm{~h}$ after labeling was $98 \%$ in sterile water and $97 \%$ in human serum.

TEM images showed that the ${ }^{99} \mathrm{~m}$ Tc-SPIONs had a mean diameter of $13 \mathrm{~nm}$ (SD, $2 \mathrm{~nm}$ ) and a spheric shape (Fig. 1). No degeneration of the polyethylene glycol coating or aggregation of the ${ }^{99 \mathrm{~m}} \mathrm{Tc}-\mathrm{SPIONs}$ was observed when images of SPIONs before labeling and after labeling were compared.

${ }^{99 \mathrm{~m}} \mathrm{Tc}$-SPIONs in an aqueous suspension exhibited an isoelectric point at $\mathrm{pH} 5.2$, lower than that exhibited by unlabeled SPIONs (pH 6.2). At $\mathrm{pH} 4$ and above $\mathrm{pH} \mathrm{6}$, the ${ }^{99 \mathrm{~m}} \mathrm{Tc}$-SPIONs had an absolute $\zeta$-potential between 5 and $15 \mathrm{mV}$. The ${ }^{99 \mathrm{~m}} \mathrm{Tc}-$ SPIONs had a stable negative charge of $-5 \mathrm{mV}$ in the $\mathrm{pH}$ range of $7-8$, like most biomolecules under physiologic conditions.

\section{Imaging}

The planar and SPECT projections were evaluated with a Xeleris Functional Imaging Workstation (GE Healthcare). The uptake of radioactivity in the lymph nodes was clearly visualized on the images (Fig. 2). The level of intensity of the ${ }^{99 \mathrm{~m}} \mathrm{Tc}-\mathrm{SPIONs}$ was highest in the popliteal lymph nodes, in good agreement with the biodistribution data.

MR images visualizing the popliteal lymph nodes are shown in Figure 3. The right popliteal lymph node was easily identified as the SLN with SE and GRE sequences. The popliteal lymph node on the left side served as a reference node. The SLN containing the ${ }^{99 \mathrm{~m} T c-S P I O N s}$ appeared to be darker than normal nodes in both SE and GRE images because of shortening of the T2 relaxation time and susceptibility effects causing signal loss. The SE images showed good delineation of the lymph nodes and good morphologic resolution; however, the GRE images were more sensitive to susceptibility effects and therefore showed susceptibility blooming artifacts caused by the iron oxide core and the high injected dose. The high-resolution SE images also revealed nonhomogeneous uptake of the contrast agent in the SLN.

\section{Biodistribution}

The amount of clearance of the ${ }^{99 \mathrm{~m}} \mathrm{Tc}-\mathrm{SPIONs}$ from the injection site was between 15\% and 33\% $4 \mathrm{~h}$ after injection. The mean uptake in the lymph nodes, kidneys, spleen, and liver is shown in Figure 4. A higher level of uptake of the ${ }^{99 \mathrm{~m} T c-S P I O N s}$ was found in the SLN $(211 \pm 225 \% \mathrm{ID} / \mathrm{g})$ than in organs such as the spleen $(0.2 \pm 0.1 \% \mathrm{ID} / \mathrm{g})$, kidneys $(0.3 \pm 0.08 \% \mathrm{ID} / \mathrm{g})$, and liver $(1.4 \pm 0.7 \% \mathrm{ID} / \mathrm{g})$. The large standard deviation in the SLN was caused by the high level of uptake $(578 \% \mathrm{ID} / \mathrm{g})$ obtained in 1 animal.

The distribution of the ${ }^{99 \mathrm{~m}} \mathrm{Tc}-\mathrm{SPIONs}$ within the popliteal and lumbar lymph nodes, as investigated with DARG revealed a high level of nanoparticle accumulation in the subcapsular and cortical sinuses but a lack of nanoparticles in the medullary cord.

\section{DISCUSSION}

The labeling method described in this work is simple and straightforward. When the oxidation state of ${ }^{99} \mathrm{~m} \mathrm{TcO}_{4}{ }^{-}$is reduced with a stannous chloride solution, ${ }^{99 \mathrm{~m}} \mathrm{Tc}$ binds to the functionalized polyethylene glycol coating. Free ${ }^{99 \mathrm{~m}} \mathrm{Tc}$ and other impurities in the form of ${ }^{99} \mathrm{~m} \mathrm{TcO}_{2}$ and ${ }^{99 \mathrm{~m}} \mathrm{Tc}$-tin
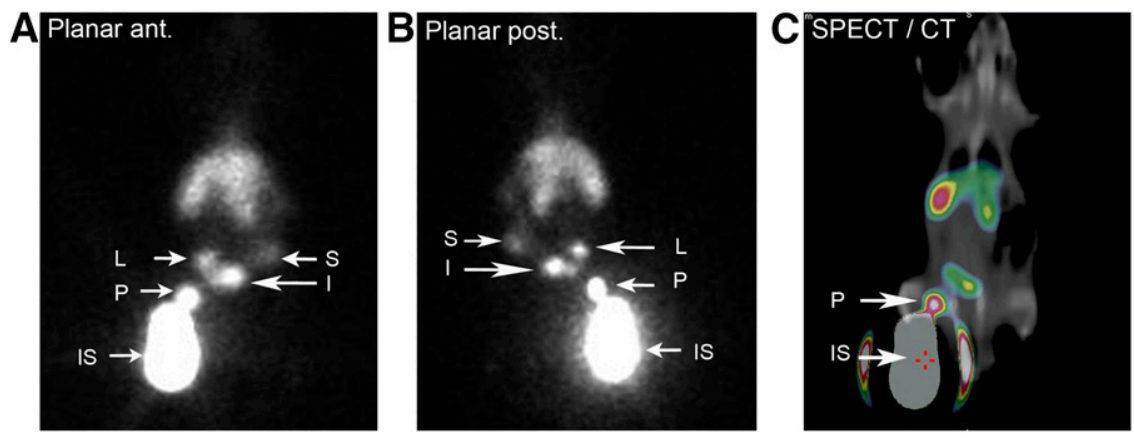

FIGURE 2. Scintillation camera and SPECT/ $\mathrm{CT}$ images of rat $4 \mathrm{~h}$ after injection with ${ }^{99 \mathrm{mTC}-}$ SPIONs. (A) Planar anterior (ant.) image. (B) Planar posterior (post.) image. (C) Fused whole-body SPECT/CT image. Arrows indicate injection site (IS) and popliteal $(P)$, iliac (I), and lumbar (L) lymph nodes. Shape of liver overlapping kidneys can be distinguished. $\mathrm{S}=$ lymph node in fatty tissue under the skin. 
FIGURE 3. Representative coronal MR images of rat visualizing SLN (thick arrow) on injection side and contralateral node (thin arrow). Accumulation of tracer is clearly visible in SLN on both SE image (A) and GRE image (B).
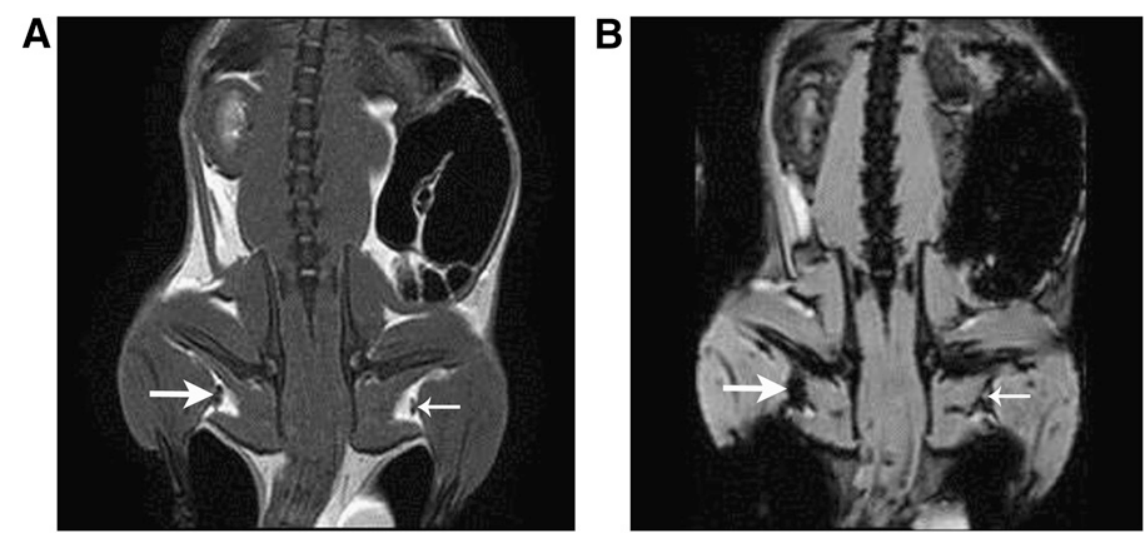

colloids may also be formed. However, it is not possible to separate colloidal impurities from the labeled compound by ITLC. Therefore, the ${ }^{99 \mathrm{~m}} \mathrm{Tc}-\mathrm{SPIONs}$ were separated from the buffer solution with MACS MS columns and a magnet, and the radioactivity was measured. The results obtained with ITLC and the magnetic separation technique were the same. This result indicates that no or only small amounts of other ${ }^{99 \mathrm{~m}} \mathrm{Tc}$-colloids were formed.

The exact sizes of SPIONs may differ, depending on the measurement techniques used. TEM, dynamic light scattering, and x-ray diffraction are 3 commonly used techniques. The advantage of TEM measurement (used in the present study) is that it allows investigation of the size, shape, and aggregation of ${ }^{99 \mathrm{~m}} \mathrm{Tc}-\mathrm{SPIONs}$ at the same time. The drawbacks are that the samples are spotted onto a grid to dry and the heat from the electron beam may affect the coating of the nanoparticles, possibly resulting in a diameter slightly smaller $(\sim 5 \mathrm{~nm})$ than the hydrodynamic diameter measured with dynamic light scattering. TEM was found to be a reliable method of measuring the sizes of SPIONs both before labeling and after labeling.

The surface properties of nanoparticles not only control colloidal stability against aggregation but also play an important role in transport by the lymphatic system and biodistribution of the contrast agent. The magnitude of the $\zeta$-potential is related to the colloidal stability of SPIONs, indicating the degree of repulsion between charged particles in dispersion. The $\zeta$-potential of colloids should be greater than $30 \mathrm{mV}$ (positive or negative) to avoid aggregation when the colloids are electrically stabilized. In the present study, the SPIONs were sterically stabilized, and a low negative or neutral $\zeta$-potential was preferable to obtain a longer half-life in vivo.

The SPECT and MR images demonstrated that ${ }^{99 \mathrm{~m} T c-}$ SPIONs can be detected with both techniques and can be used as multimodality tracers or contrast agents for SLN imaging. A small amount of ${ }^{99 \mathrm{~m}} \mathrm{Tc}-\mathrm{SPIONs}$ was transported by the lymph through other nodes and accumulated in the liver and spleen. Both imaging techniques were able to successfully identify the SLN in rats. MR images provide important anatomic information and good soft-tissue con- trast, allowing analysis of the size and shape of the SLN, whereas SPECT has high sensitivity, so that even a small amount of a contrast agent can be detected.

The clearance of ${ }^{99 \mathrm{~m} T c-S P I O N s}$ from the injection site depends not only on the size, shape, charge, and concentration of a contrast agent but also on the injection technique. The reproducibility of subcutaneous injections is important because minor deviations can lead to considerable differences in uptake, as seen in 1 of the 5 animals in the present study. The amount of clearance of the contrast agent from the injection site (15\%-33\%) in the present study was comparable to that reported for similarly sized nanocolloids by Bergqvist et al. (13) and Bengele et al. (14).

Because the MR images (SE sequence) revealed a nonhomogeneous accumulation of ${ }^{99 \mathrm{~m}} \mathrm{Tc}-\mathrm{SPIONs}$ in normal nodes, further studies were conducted with DARG. The DARG images demonstrated an uptake pattern suggesting that the nanoparticles not only were restrained mechanically in the lymph nodes but even were phagocytized by cells (macrophages) in the subcortical, cortical, and medullary sinuses. Similar results were reported by Lee et al. (15). The medullary cord showed no signal on the DARG images because of the lack of radiolabeled nanoparticles.

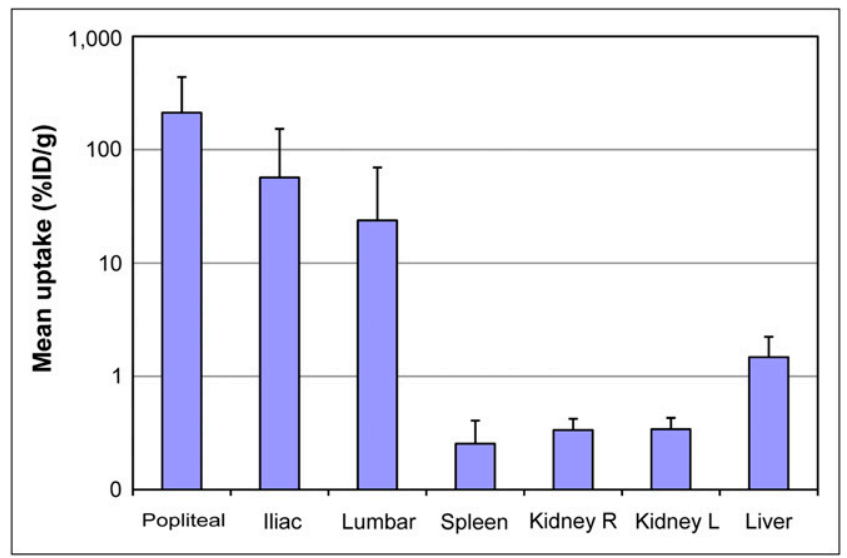

FIGURE 4. Biodistribution of ${ }^{99 \mathrm{~m} T c-S P I O N s}$ in 5 white Wistar rats $4 \mathrm{~h}$ after injection. Uptake is shown as mean \pm SD \%ID/g. Level of uptake in inguinal and other lymph nodes was less than $0.07 \% \mathrm{ID} / \mathrm{g}$. 
This information is important for understanding the different patterns of contrast-enhanced normal nodes and nodes with metastatic involvement (in future studies), guiding the development of targeting strategies, and performing detailed dosimetry calculations.

\section{CONCLUSION}

The present study demonstrated the feasibility of labeling SPIONs with ${ }^{99 \mathrm{~m}} \mathrm{Tc}$. The accumulation of ${ }^{99 \mathrm{~m}} \mathrm{Tc}-$ SPIONs in lymph nodes after subcutaneous injection in rats, verified by SPECT/MRI detection, is encouraging for future applications in breast cancer and malignant melanoma.

\section{DISCLOSURE STATEMENT}

The costs of publication of this article were defrayed in part by the payment of page charges. Therefore, and solely to indicate this fact, this article is hereby marked "advertisement" in accordance with 18 USC section 1734.

\section{ACKNOWLEDGMENTS}

This study was performed with generous support from the Swedish Cancer Foundation, the Swedish Science Council, Mrs. Berta Kamprad's Foundation, Gunnar Nilsson's Foundation, and the ALF Foundation of the Medical Faculty of Lund University. The SPIONs were provided by Genovis AB, Lund, Sweden. The authors thank Anders Örbom (Department of Medical Radiation Physics, Lund University) for help with the DARG measurements, Eric Carleman (Lund University Bioimaging Center) for help with the TEM images, and Thuy Tran (Lund University Bioimaging Center) for comments on the article. Sarah
Fredriksson, Fredrik Olsson, Pontus Kjellman, and Genovis $\mathrm{AB}$ have no commercial interest in and have not provided any financial support for the submitted work. No other potential conflict of interest relevant to this article was reported.

\section{REFERENCES}

1. Schauer AJ, Becker W, Reiser M, Possinger K. The Sentinel Lymph Node Concept. Berlin, Germany: Springer-Verlag Berlin Heidelberg; 2005.

2. Buscombe J, Paganelli G, Burak ZE, et al. Sentinel node in breast cancer procedural guidelines. Eur J Nucl Med Mol Imaging. 2007;34:2154-2159.

3. Strand SE, Bergqvist L. Radiolabeled colloids and macromolecules in the lymphatic system. Crit Rev Ther Drug Carrier Syst. 1989;6:211-238.

4. Strand SE, Jönsson PE, Bergqvist L, et al. Preoperative ${ }^{99 m} \mathrm{Tc}$-antimony sulphide colloid scintigraphy for identification of the lymph drainage in patients with malignant melanoma. Progress in Radiopharmacology. 1981;2:293-304.

5. Wilhelm AJ, Mijnhout GS, Franssen EJ. Radiopharmaceuticals in sentinel lymph-node detection: an overview. Eur J Nucl Med. 1999;26(4 suppl):S36-S42.

6. Jain R, Dandekar P, Patravale V. Diagnostic nanocarriers for sentinel lymph node imaging. J Control Release. 2009;138:90-102.

7. Burns DL, Mascioli EA, Bistrian BR. Parenteral iron dextran therapy: a review. Nutrition. 1995;11:163-168.

8. Ferrucci JT, Stark DD. Iron oxide-enhanced MR imaging of the liver and spleen: review of the first 5 years. AJR. 1990;155:943-950.

9. Bellin MF, Beigelman C, Precetti-Morel S. Iron oxide-enhanced MR lymphography: initial experience. Eur J Radiol. 2000;34:257-264.

10. Russell M, Anzai Y. Ultrasmall superparamagnetic iron oxide enhanced MR imaging for lymph node metastases. Radiography. 2007;13:73-84.

11. Harisinghani MG, Saksena MA, Hahn PF, et al. Ferumoxtran-10-enhanced MR lymphangiography: does contrast-enhanced imaging alone suffice for accurate lymph node characterization? AJR. 2006;186:144-148.

12. Wingårdh $\mathrm{K}$, Strand SE. Evaluation in vitro and in vivo of two labelling techniques of different ${ }^{99 \mathrm{~m}} \mathrm{Tc}$-dextrans for lymphoscintigraphy. Eur J Nucl Med. 1989;15:146-151.

13. Bergqvist L, Strand SE, Persson BR. Particle sizing and biokinetics of interstitial lymphoscintigraphic agents. Semin Nucl Med. 1983;13:9-19.

14. Bengele HH, Palmacci S, Rogers J, et al. Biodistribution of an ultrasmall superparamagnetic iron oxide colloid, BMS 180549 , by different routes of administration. Magn Reson Imaging. 1994;12:433-442.

15. Lee AS, Weissleder R, Brady TJ, Wittenberg J. Lymph nodes: microstructural anatomy at MR imaging. Radiology. 1991;178:519-522. 